\title{
Sensitivität und Selektivität optischer und neuartiger MEMS-Wasserstoffsensoren
}

\author{
Marion Wienecke1, Laura Godenrath ${ }^{2}$, Bernd Zacharias ${ }^{2}$ \\ ${ }^{1}$ Hochschule Wismar, Institut für Oberflächen- und Dünnschichttechnik, \\ PF1210, 23952 Wismar, Deutschland \\ ${ }^{2}$ Materion $\mathrm{GmbH}$, Alter Holzhafen 15, 23966 Wismar, Deutschland
}

\section{Zusammenfassung}

In diesem Beitrag werden die Potenziale von optisch schaltenden Pd-Dünnfilmsensoren (POS) untersucht sowie neuartige mikro-elektro-mechanischen Systeme (MEMS), die die Volumenänderung bei der Hydrierung des Pd ausnutzen. Beide Systeme können Wasserstoffkonzentrationen von 0,1 bis 100 vol \% sicher und reproduzierbar nachweisen. Das physikalische Schalten von Pd bei der Einlagerung von Wasserstoff ist frei von Querempfindlichkeiten. Die Ansprechzeiten betragen unter $30 \mathrm{~s}$ (optischer Sensor) bzw. $5 \mathrm{~s}$ (MEMS-Sensor). Mit Pd-basierten MEMS-Sensoren können minimale Nachweisgrenzen von 100 ppm erreicht werden. Es werden mögliche Einsatzgebiete dieser Sensoren diskutiert.

Keywords: Palladium, optischer Wasserstoffsensor, MEMS-Wasserstoffsensor, Ansprechzeit, Querempfindlichkeit

\section{Motivation}

Seit mehr als 100 Jahren, seit Wasserstoff technisch genutzt wird, besteht die Notwendigkeit Wasserstoffkonzentrationen nachzuweisen und verlässlich zu messen. Für viele Industriezweige haben sich inzwischen Wasserstoffsensoren etabliert, so z.B. in der chemischen Industrie, in der Metallurgie, im Bergbau, bei der Überwachung von Kernkraftwerken. Die physikalisch-chemischen Grundlagen, auf denen diese Sensoren beruhen, sind seit vielen Jahrzehnten gut untersucht [1]. Eine Vielzahl von marktverfügbaren $\mathrm{H}_{2}$-Sensoren beruht auf einigen wenigen Messprinzipien, darunter hauptsächlich elektro-chemische Sensoren, katalytische Pellistoren, MetallOxid Sensoren, Wärmeleitsensoren, MetallOxid-Halbleiter-Sensoren. Diese Sensoren dienen überwiegend zur Kontrolle und zum Explosionsschutz von stationären Anlagen und erfüllen entsprechende Standards. Nach ISO 26142 sind dies folgende Anforderungen [2]:

- Messbereich bis 4 vol\% in Luft, Überlebensfähigkeit bis 100 vol\%,

- Nachweisgrenze 100 ppm,

- Ansprechzeit (T90): $30 \mathrm{~s}$

- Erholungszeit (T10): $60 \mathrm{~s}$,

- Umgebungstemperatur: -20 bis $+50^{\circ} \mathrm{C}$,

- Umgebungsdruck: 80-110 kPa,

- Feuchtigkeit: $20-80 \%$ relative Feuchte,

- Messgenauigkeit: \pm 25 oder $50 \%$ abhängig von der $\mathrm{H}_{2}$-Konzentration.
Spezifische Anforderungen ergeben sich für Anwendungen zur Prozesskontrolle sowie insbesondere für neue Anwendungsfelder im Bereich der erneuerbaren Energien [2], z.B. zur Überwachung von Brennstoffzellen, im Automobilbereich, bei der Einspeisung von regenerativ erzeugtem Wasserstoff in Erdgasleitungen, sowie auch in der Medizintechnik zur Messung von $\mathrm{H}_{2}$-Anteilen im Atemgas, z.B. zum Nachweis von Laktoseintoleranz [3]. Diese neuen Anforderungen beziehen sich vor allem auf einen erweiterten Messbereich, für die Überwachung von mobilen Brennstoffzellen z.B. bis Wasserstoffkonzentrationen von 100 vol\%, schnellere Ansprechzeiten, im Automobilbereich z.B. kleiner als $1 \mathrm{~s}$ und im Medizintechnikbereich deutlich geringere Nachweisgrenzen von weit unter 100 ppm. Ein Nachteil der o.g. marktverfügbaren $\mathrm{H}_{2}-$ Sensoren, insbesondere für die erwähnten neuen Einsatzgebiete, besteht zudem darin, dass diese alle, außer WLD-Sensoren, mehr oder weniger starke Querempfindlichkeiten zu anderen brennbaren Gasen aufweisen [4]. Seit jüngerer Zeit sind $\mathrm{H}_{2}$-Sensoren auf dem Markt, die auf dem physikalischen Schalten von $\mathrm{Pd}$ oder Pd-Legierungen beruhen und von daher frei von Querempfindlichkeiten sind. Dazu gehören optische, resistive und MOS-Sensoren mit Pd-basierten Dünnfilmen [5]. Weitere neue Herausforderungen beziehen sich auf die Akzeptanz in den genannten Einsatzfeldern, da 
etwa in der regenerativen Energiegewinnung, für mobile Geräte und im Automobilbereich und erst recht in der Medizintechnik die Messsysteme dezentral, nahe am Endverbrauer zum Einsatz kommen. In diesem Zusammenhang bestehen neue Anforderungen hinsichtlich der Sicherheit, des Energieverbrauchs, der Miniaturisierung und des Preises. Auch in dieser Hinsicht weisen Pdbasierte Dünnfilmsensoren Potenziale auf, da sie mittels Siliziumtechnologie in einer Massenfertigung hergestellt werden können.

In diesem Beitrag werden die Potenziale von optisch schaltenden Pd-Dünnfilmsensoren (POS) untersucht sowie neuartige mikroelektro-mechanischen Systeme (MEMS), die die Volumenänderung bei der Hydrierung des Pd ausnutzen.

\section{Material und Methoden}

\section{1. physikalische Eigenschaften des $\mathrm{Pd}-\mathrm{H}$ Systems}

Die schaltbaren physikalischen Eigenschaften beruhen auf dem Effekt der atomaren Einlagerung von Wasserstoff auf Zwischengitterplätzen im Metallgitter. In Abhängigkeit von seiner Konzentration wird der Wasserstoff beim Auftreffen auf Metall zunächst adsorbiert, dissoziiert und durch Diffusion im Metallgitter gelöst. Die a-Phase, eine feste Lösung, entsteht. Bei Überschreitung einer bestimmten metallspezifischen Wasserstoffkonzentration wird die Hydridphase (á-Phase oder oft als $\beta$-Phase bezeichnet) gebildet. Dieser Prozess läuft schon bei Raumtemperatur ab und ist reversibel. Im Palladium besteht die a-Phase bei Raumtemperaturen bis zu einer Wasserstoffkonzentration von 1,68 at\%. Im Konzentrationsbereich von 1,68 bis 37,6 at $\%$ ist das Stoffsystem zweiphasig und besteht aus der $\alpha$ und der $\beta$-Phase. Ab 37,6 at\% ist nur noch die $\beta$-Phase zu finden [6]. Die Löslichkeit von Wasserstoff in Palladium sinkt mit steigender Temperatur und steigt mit steigendem $\mathrm{H}_{2}-$ Partialdruck. Die a-Phase ist metallisch leitfähig und opak. Der Widerstand steigt mit zunehmender Konzentration von $\mathrm{H}$ im Metallgitter aufgrund von durch die Einlagerung bedingter Elektronenstreuung. Die $\beta$-Phase ist halbleitend und transparent [7].

Während der Wasserstoffeinlagerung und insbesondere beim Phasenwechsel kommt es zu einer Volumenänderung. Die Gitterkonstante für reines $\mathrm{Pd}$ beträgt $3,887 \AA$, bis zum Wasserstoffgehalt von ca. 2 at\% steigt sie auf $3,895 \AA$ und für die $\beta$-Phase mit ca. 37,6 at $\%$ Wasserstoff beträgt die Gitterkonstante $0,402 \AA$ [8]. Damit ist eine Volumenausdehnung von $3,5 \%$ verbunden [6].
Diese Volumenausdehnung folgt der Formel

$$
\Delta \mathrm{V} / \mathrm{V}=\Delta \mathrm{V} / \mathrm{\omega} \mathrm{C}_{\mathrm{H}}
$$

$\Delta \mathrm{a} / \mathrm{a}=1 / 3 \Delta \mathrm{v} / \mathrm{\omega} \mathrm{CH}_{\mathrm{H}}$

$\Delta a / a=a_{H} C_{H}$

$\Delta \mathrm{V} \quad$ - Volumenwechsel des Metalls bei Lösung von $n$ Wasserstoffatomen

$\mathrm{V} \quad$ - Ausgangsvolumen des Metalls

$\Delta v \quad$ - charakteristische Volumenwechsel pro Wasserstoffatom, $\Delta \mathrm{v}$ steht in direkter Beziehung zum molaren Volumen $\left(\mathrm{V}_{\mathrm{M}}=\Delta \mathrm{v} \cdot \mathrm{L}\right.$, L- Avogadro Konstante

$\omega \quad$ - Atomvolumen eines Metallatoms

$\mathrm{CH}_{\mathrm{H}} \quad$ - Verhältnis n/N, Verhältnis der Anzahl der Wasserstoffatome zur Anzahl der Metallatome (bei 50 at $\%$ beträgt $\mathrm{CH}_{\mathrm{H}}=1$ )

a - Gitterkonstante,

$\alpha_{H} \quad$ - Ausdehnungskoeffizient bezüglich $\mathrm{H}$ Einlagerung, beträgt für $\mathrm{Pd} 0,063$

Diese Volumenänderung des Pd ist die Ursache für mangelnde Haftfestigkeit von PdDünnfilmen. Strategien, diese zu verbessern bestehen darin, das $\mathrm{Pd}$ zu legieren oder haftvermittelnde Schichten anzuwenden, wir verweisen auf Fedtke et al. und dort zitierte Autoren [9].

Die Wasserstoffkonzentration in der $\alpha$-Phase ist eine streng Partialdruckabhängige Funktion. Die Einlagerung von Wasserstoff im Metall erfolgt proportional der Wurzel aus dem Wasserstoffpartialdruck (Sievertsches Gesetz).

$$
\mathrm{C}_{\mathrm{H}} \approx \mathrm{p}\left(\mathrm{H}_{2}\right)^{1 / 2} / \mathrm{K}
$$

$\mathrm{p}\left(\mathrm{H}_{2}\right)$ - Wasserstoffpartialdruck

$\mathrm{K}$ - temperaturabhängige Sievertskonstante, für $\mathrm{Pd}=0,12$ bei $300 \mathrm{~K}$

\subsection{Sensitivität und Selektivität Pd-basierte Wasserstoffsensoren}

Pd-basierte Wasserstoffsensoren sind heute marktverfügbar und werden mit verschiedenen Messprinzipien angeboten.

Der Nachweis der Wasserstoffkonzentration erfolgt optisch meist über die Bestimmung der Änderung der Transmission oder Reflexion des Pd-dünnfilmes mittels Transmissions- bzw. Reflexionsmessungen. Erstmalig wurde dies 1993 von Oh et al. berichtet [10]. Mehr sensitive aber aufwendigere Verfahren zur Bestimmung der Änderung der optischen Eigenschaften sind Surface plasmon resonance und interferometrische Messungen, Fiber-Bragg-Gitter sowie Messungen der Evaneszenz an Lichtleitern [5]. 
Resistive Pd-basierte Sensoren nutzen den um bis zu $80 \%$ steigenden Widerstand der Dünnfilme beim Übergang von der $\alpha$ - zur $\beta$ Phase als Maß für die Wasserstoffkonzentration. Diese Sensoren können ebenso wie die optische Sensoren Wasserstoffkonzentrationen bis $100 \%$ messen. Weiterhin kommen MOS-Sensoren mit Pd-Metall als Gate-Elektrode zum Einsatz und die Kapazität der MOSFET als Folge des steigenden Widerstands am Gate ist das Maß für die Wasserstoffkonzentration. Diese Sensoren können kleine Wasserstoffkonzentrationen nachweisen, sie beruhen darauf, dass die metallische $\alpha$-Phase ihren Widerstand mit steigender Wasserstoffeinlagerung ändert. Eine Übersicht über die Prinzipien und Messbereiche von marktverfügbaren $\mathrm{Pd}$ basierten Wasserstoffsensoren zeigt Tabelle 1.

Tab. 1: $\quad$ Übersicht marktverfügbarer $P d$ basierter Wasserstoffsensoren [5]

\begin{tabular}{|c|c|c|}
\hline Hersteller & Messprinzip & Messbereich \\
\hline H2scan & resistiv & $0,5-100 \mathrm{vol} \%$ \\
\hline H2scan & kapazitiv & $15 \mathrm{ppm}-1 \mathrm{vol} \%$ \\
\hline Makel & resistiv & $0,1-100 \mathrm{vol} \%$ \\
Engineering & & \\
\hline Applied & Resonanz & $100 \mathrm{ppm}-$ \\
Nanotech & & $5 \mathrm{vol} \%$ \\
\hline
\end{tabular}

Betrachtet man in referierten Fachzeitschriften Angaben zur Sensititvität und Ansprechzeit von $\mathrm{Pd}$-basierten Sensorsystemen so zeigt sich, dass Ansprechszeiten von unter $3 \mathrm{~s}$ nicht erreicht werden. Boon-Brett berichtet in einem Review Artikel Ansprechzeiten ( $\left.T_{90}\right)$ von $>30 \mathrm{~s}$ für optische $\mathrm{H}_{2}$-Sensoren [2]. Liu etal. [11] zeigen für optische, auf Transmission beruhende Sensoren für 4 vol\% Wasserstoff eine Ansprechszeit von wenigen Sekunden. Die Ansprechzeit ist invers proportional zur Wasserstoffkonzentration, je kleiner die Konzentration, desto langsamer der Sensor. Für kleine Konzentrationen zwischen 10 und 100 ppm berichtet Lim [12] 2 min bis $30 \mathrm{~s}$ Ansprechzeit. Untersucht wurden hier mittels Mikrosystemtechnik hergestellte freitragende nanoskalige $\mathrm{Pd} / \mathrm{C}$-Nanodrähte.

Bezüglich der unteren Nachweisgrenze wird übereinstimmend berichtet, dass nanoskalige Schichtdicken und Kristallitgrößen sowie nanoskalig strukturierte Trägermaterialien zu höherer Sensitivität führen: Su et al. [13] berichten über resistive Messungen an CNT getragene nanoskaligen Pd-Strukturen und erreichen 200 ppm als untere Nachweisgrenze. Über Graphene getragene Strukturen berichten Panday et al. [14] und zeigen in resistiven Messungen minimale Nachweiskonzentration ebenfalls von 200 ppm. Die Ansprechzeiten in beiden Berichten liegen bei mehreren Minuten. Eine systematische Untersuchung von nanoskaligen Pd-Strukturen zeigen Noh et al. [15]. Es werden 2-D Palladium Dünnfilme, 1-D(Nanowire)-Sensorstrukturen und Nanopartikel (0-D) in resistiven Messungen untersucht. Es wird gezeigt, dass je nanoskaliger die Strukturen desto kürzer wird die Ansprechzeit. Es werden minimale Nachweisgrenzen von 100 ppm und Ansprechzeiten zwischen 10 und $30 \mathrm{~s}$ erreicht.

Untersuchungen der Selektivität von Pdbasierten Sensoren in internationalen Fachzeitschriften zeigen übereinstimmend, dass $\mathrm{Pd}$-basierte $\mathrm{H}_{2}$-Sensorstrukturen unabhängig vom Messprinzip keine Querempfindlichkeiten $z u$ anderen für die o.g. Anwendungen relevanten Gasen aufweisen. Vergleichende Untersuchungen von marktverfügbaren Wasserstoffsensoren sind $z$.B. von Palmisano et al. [4] durchgeführt worden. PdDünnfilmsensoren zeigen demnach keine Querempfindlichkeit zu 1 vol\% $\mathrm{CH}_{4}, \mathrm{CO}_{2}$ und 50 ppm CO. Panday et al. [14] zeigen für resistive Messungen an Graphene getragenen Pd-NanoDünnfilmen, dass gegen $\mathrm{CO}$, Ethanol und Toluol keine Querempfindlichkeiten bestehen.

\section{3. optisch schaltende Wasserstoffsensoren}

In dieser Untersuchung haben wir PE-Folien beidseitig (siehe Abbildung 1) mit wenigen Nanometer dicken Pd/Au-Legierungen besputtert (Magnetron-Sputtern mit Patchworktarget). Die Au-Anteile wurden variiert von 18 bis 64 at\%. Die Schichtdicken betrugen zwischen 10 und $15 \mathrm{~nm}$. Gemessen wurde die Transmission von mono-chromatischem Licht mit einer Wellenlänge von $650 \mathrm{~nm}$. Hinsichtlich der verwendeten Messtechnik verweisen wir auf Bramann et al. [16].

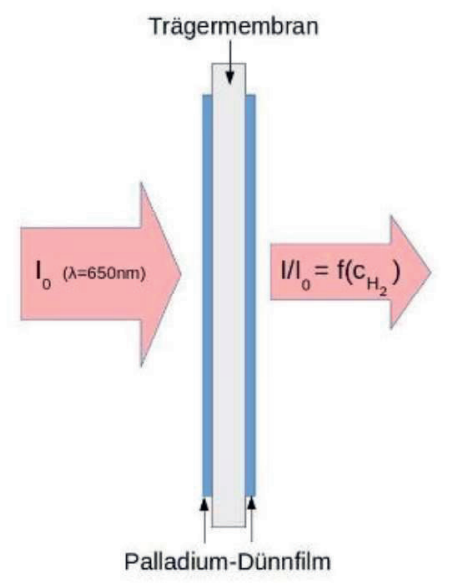

Abb. 1: Messprinzip optischer $\mathrm{H}_{2}$-Sensor, Intensität des transmittierten Lichtes mit $650 \mathrm{~nm}$ Wellenlänge ist Funktion der $\mathrm{H}_{2}$-Konzentration 


\subsection{MEMS basierte Wasserstoffsensoren}

Mikro-elektro-mechanische Sensorstrukturen zum Nachweis von Wasserstoff nutzen physikalisch die Volumenänderung des Pd oder von Pd-Legierungen bei der Einlagerung von Wasserstoff. Die Messtechnik zum Nachweis der Volumenänderung kann unterschiedlich sein. Baselt et al. [17] berichten über einen Mikrocantilever, der ähnlich wie ein Bimetallstreifen von einer Seite mit Pdbeschichtet, sich unter $\mathrm{H}_{2}$-Einfluß verformt. Diese Verformung wird kapazitiv gemessen und erlaubt den Nachweis von 1000 ppm bis 100\% Wasserstoff. Es wird für $1 \mathrm{vol} \%$ Wasserstoff eine Ansprechzeit von $90 \mathrm{~s}$ angegeben.

Gurusamy et al. [18] beschreiben ebenfalls einen Microcantilever. Es können 50 ppm $\mathrm{H}_{2}$ durch optische Auslesung der Verformung des Cantilevers sicher nachgewiesen werden.

Lee et al. [19] beschreiben Design-Vorschläge für sogenannte Nanogap-basierte Sensoren. Sie beruhen auf resistiven Messungen an $\mathrm{Pd}$ Nano-Strukturen, die bei Volumenausdehnung perkolieren und dadurch leitfähiger werden. Für eine einfache on-off Anordnung wurde gezeigt, dass 100 ppm $\mathrm{H}_{2}$ sicher nachgewiesen werden können.

In dieser Arbeit haben wir marktverfügbare MEMS-Drucksensoren mit $\mathrm{Pd} / \mathrm{Au}$ Dünnfilmen beschichtet $(100 \mathrm{~nm}$, Magnetron-Sputtern mit Patchworktarget). Eine Prinzipskizze zeigt Abbildung 2. Die MEMS-Drucksensoren beruhen auf piezoresistiven Eigenschaften von Silizium. Widerstandsstrukturen in Form einer Wheatstone-Brücke werden verstimmt, wenn die Si Membran sich verformt. MEMSDrucksensoren werden in Massenfertigung mittels Siliziumtechnologie hergestellt. Das Ausgangssignal des MEMS-Sensors wird in den nachfolgenden Abbildungen als Maß für die Wasserstoffkonzentration herangezogen.
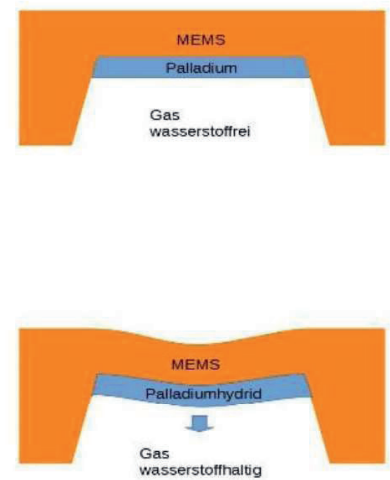

Abb. 2: Sensorprinzip eines Pd modifizierten MEMS-Sensors

\section{Ergebnisse}

3.1. Sensitivität und Selektivität optischer Wasserstoffsensoren

Abbildung 3 zeigt Messungen der optischen Transmission an einer oben beschriebenen Struktur (Abbildung 1) im Konzentrationsbereich 1 bis 100 vol\% Wasserstoff in Luft. Dargestellt ist ein Messzyklus für $\mathrm{Pd} / \mathrm{Au}$ mit 18 at\% Au. Es zeigte sich, dass Filme mit höherem Au-Anteil weniger reproduzierbare Messergebnisse und insbesondere eine starke Drift der Basislinie durch Alterungserscheinungen zeigen.

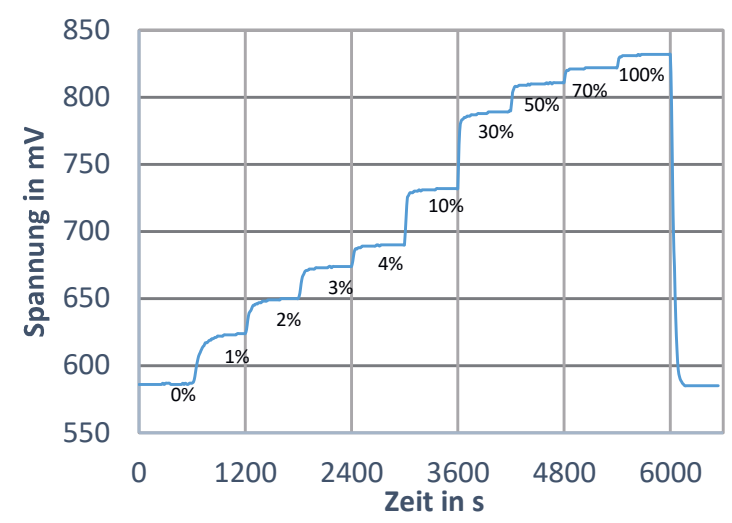

Abb. 3: Messung Transmission von Licht für H2-Konzentrationen 1 bis 100 vol\% in Luft, Pd/Au Dünnfilm mit 18 at\% Au

In Abbildung 4 ist die Sensorkennlinie in logarithmischer Form dargestellt. Die Transmission als Maß für das Verhältnis der $\mathrm{H} / \mathrm{Pd}$ Atome im Kristallgitter folgt der Wurzel des $\mathrm{H}_{2}$-Partialdruckes (Gleichung 4), sodass in lorarithmischer Darstellung ein linearer Verlauf entsteht.

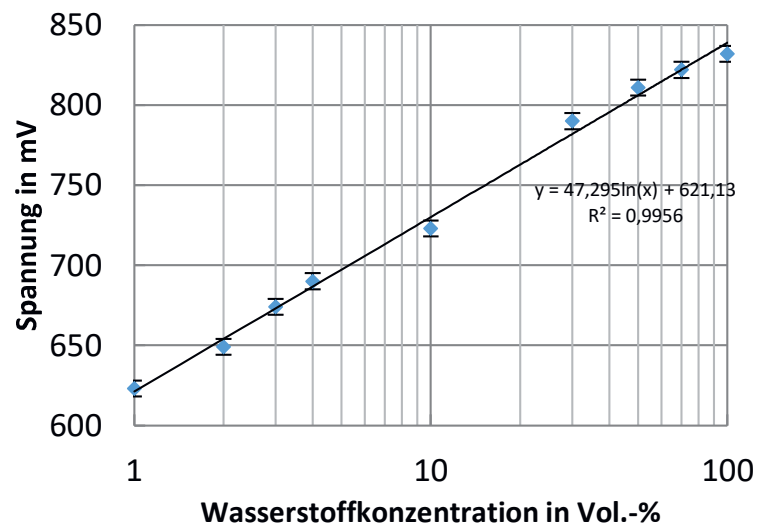

Abb. 4: Kennlinie optischer H2-Sensor aus Messung in Abbildung 3 
Abbildungen 5 zeigt im Detail den Zeitverlauf des Signals beim Sprung von 3 auf 4 vol\% Wasserstoff bei der optischen Messung. Es zeigt sich eine T$_{90}$ Ansprechzeit von $20 \mathrm{~s}$.

Messungen der optischen Transmission unter Wasserstoffeinfluss wurden auch für kleinere Konzentrationen zwischen 1000 und 5000 ppm durchgeführt. Konzentrationsverläufe lassen sich sicher und reproduzierbar messen bis etwa 1000 ppm als untere Nachweisgrenze. Abbildung 6 zeigt im Detail die Abklingzeit auf $10 \%$ des Ausgangswertes ( $\left.T_{10}\right)$ beim Sprung von 5000 auf 1000 ppm Wasserstoff in Luft. Die $\mathrm{T}_{10}$-Zeit beträgt ebenfalls etwa $20 \mathrm{~s}$.

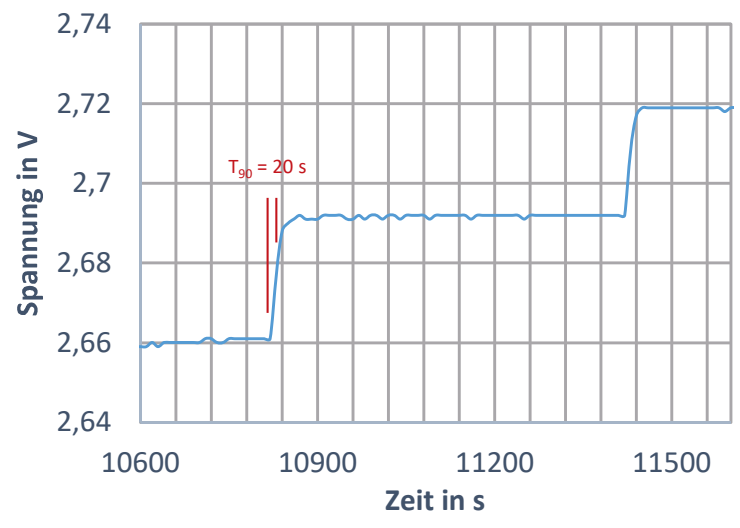

Abb. 5: Messung Transmission von Licht beim Wechsel von 2 auf 3 vol\% $\mathrm{H}_{2}$ Konzentration in Luft

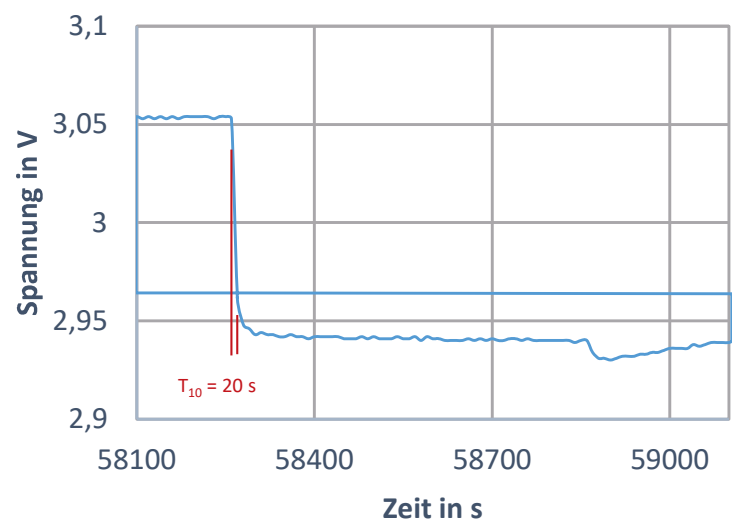

Abb. 6: Messung Transmission von Licht beim Wechsel von 5000 auf 1000 ppm $\mathrm{H}_{2}$ Konzentration in Luft

Erste Untersuchungen zur Querempfindlichkeit wurden mit der beschriebenen Sensorstruktur durchgeführt, indem der Sensor $\mathrm{CH}_{4}$-LuftGemischen anstelle von Wasserstoff-Luft ausgesetzt wurde. Eine entsprechende Messung mit $\mathrm{CH}_{4}$ Konzentrationen stufenweise von 0 auf 4 vol \% zeigt Abbildung 7. Der optische Pd-Dünnfilmsensor (blaue Kurve) zeigt darauf keine Messignale.

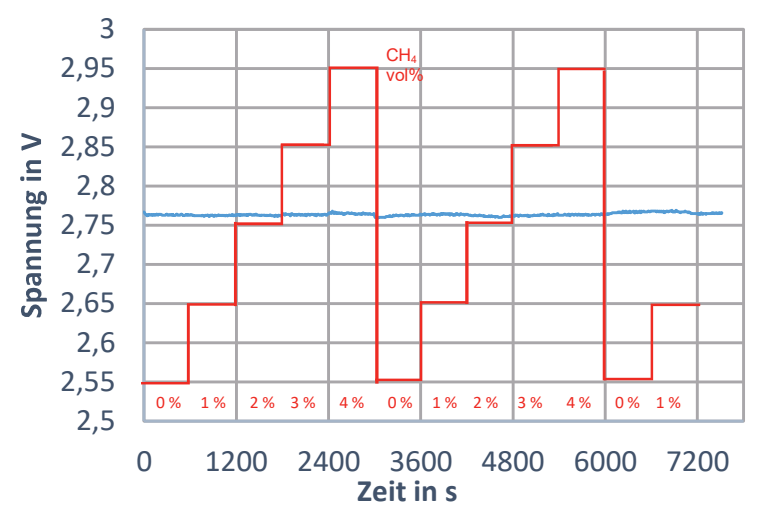

Abb. 7: Messung Transmission von Licht (blaue Kurve) beim Wechsel von $\mathrm{CH}_{4}$ Konzentrationen zwischen 0 und $4 \mathrm{vol} \%$ in Luft

\subsection{MEMS basierte Wasserstoffsensoren}

In Abbildung 8 sind mehrere Messzyklen mit wechselnden $\mathrm{H}_{2}$-Konzentrationen zwischen 1 und 10 vol \% in Luft dargestellt, gemessen mit der Pd-modifizierten MEMS-Sensorstruktur (siehe Abbildung 2). Jeder Zyklus besteht aus 10 Stufen mit 10 min Haltezeit. Es zeigt sich nach kurzem Einschwingen der MEMS Struktur ein reproduzierbarer Signalverlauf. Abbildung 9 zeigt eine Detailansicht aus weiteren zyklischen Messungen an der gleiche MEMS-SensorStruktur beim Sprung von 1 auf 10 vol\% Wasserstoff in Luft. Die T90 Zeit beträgt $5 \mathrm{~s}$.

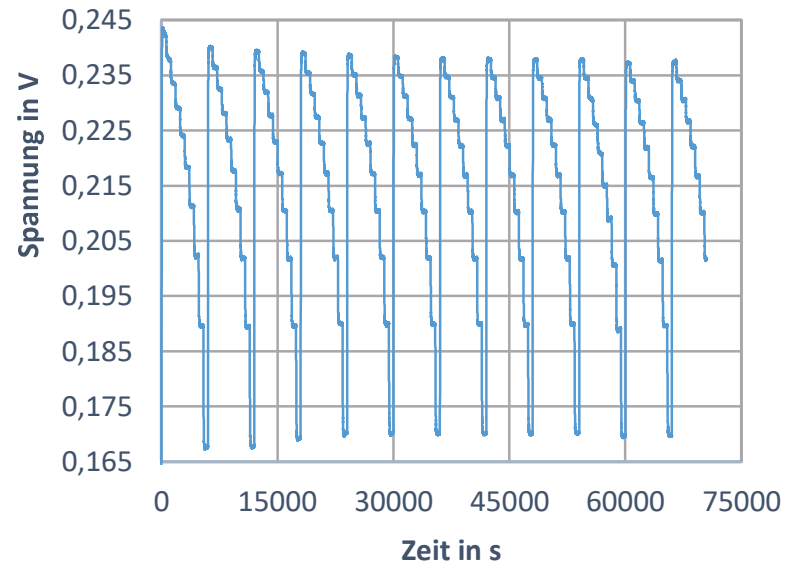

Abb. 8: Messsignal Pd-beschichteter MEMS Sensor, zyklische Messungen von 1 10 vol\% und stufenweise Absenkung in $1 \%$ Schritten von 10 auf $1 \mathrm{vol} \%$ Wasserstoff in Luft 


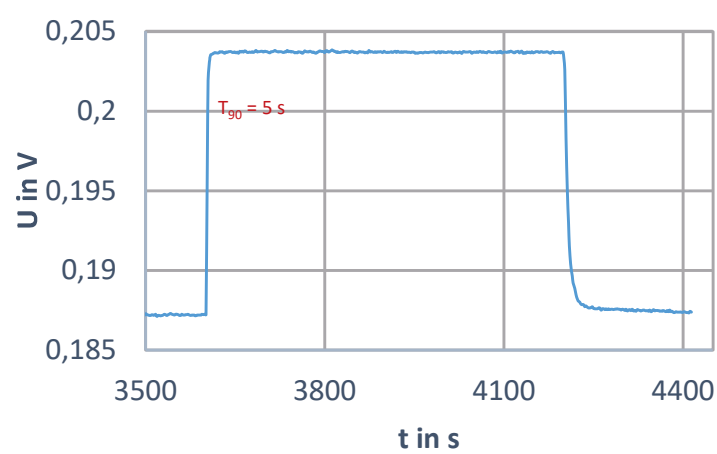

Abb. 9: Pd-modifizierter MEMS-Sensor beim Wechsel von 1 auf $10 \mathrm{vol} \%$ Wasserstoff in Luft, Messung bei Raumtemperatur

In Abbildung 10 ist ein Messzyklus über $22 \mathrm{~h}$ dargestellt mit Wechsel der $\mathrm{H}_{2}$-Konzentration von 0 auf 10 vol\% und zurück auf 0 vol\% in Luft nach jeweils $10 \mathrm{~min}$. Die Messungen erfolgten thermostatiert bei $60^{\circ} \mathrm{C}$. Die Ansprechzeit $\mathrm{T}_{90}$ beträgt jeweils wenige Sekunden. Es zeigt sich ein stabiler Signalverlauf mit konstanter Signalhöhe und eine Drift der Nulllinie. Es wurde ein Referenz MEMS Sensor gleichzeitig aufgezeichnet, der keine Pd-Beschichtung aufweist. Demnach ist die Signaldrift durch die Umgebung bedingt. Infrage kommt die Umgebungstemperatur, z.B. Tag-Nacht Rhythmus, möglicherweise ist die Thermostatierung nicht konstant. Die Abweichungen betragen weniger als $10 \%$.

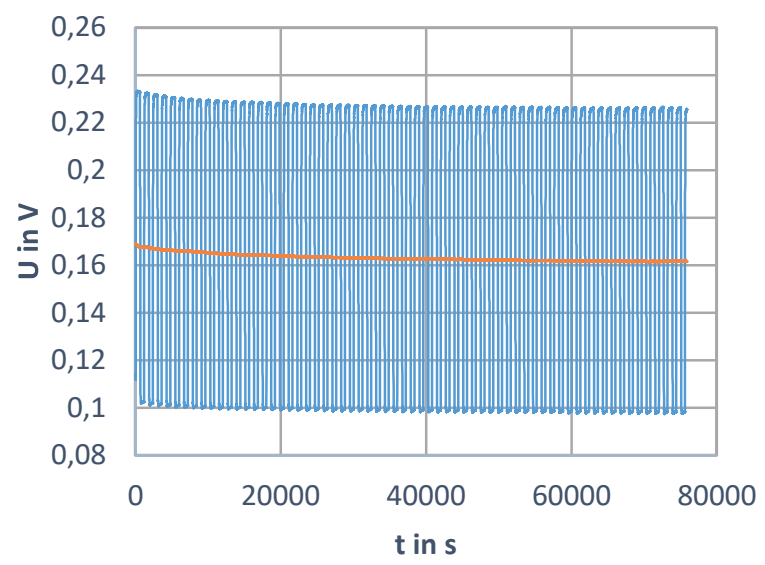

Abb. 10: Messsingnal Pd- beschichteter MEMS Sensor (blaue Kurve), zyklischer Wechsel zwischen 0 und $10 \mathrm{vol} \% \mathrm{H}_{2}$ Konzentration in Luft, thermostatiert bei $60{ }^{\circ} \mathrm{C}$, zum Vergleich unbeschichteter MEMSReferenzsensor (rote Kurve)
Untersucht wurden auch Konzentrationen unter 1000 ppm bis 100 ppm. Abbildung 11 zeigt mehrere Zyklen mit Wechsel von 400 ppm auf $1000 p p m$ und in 200 ppm Stufen zurück auf 400 ppm, gemessen mit dem gleichen $\mathrm{Pd}$ modifizierten MEMS-Sensor.

400 ppm lassen sich sicher nachweisen. Jedoch beträgt die $T_{90}$ Zeit hier beim Sprung von 400 auf $1000 \mathrm{ppm}$ etwa $3 \mathrm{~min}$. In weiteren Messungen konnten auch 100 ppm sicher nachgewiesen werden. Durch weitere Messungen mit zyklischen Wechseln der Wasserstoffkonzentration zwischen 0 und 100 ppm haben wir $T_{90}$ und $T_{10}$ Zeiten von mehr als 5 min festgestellt.

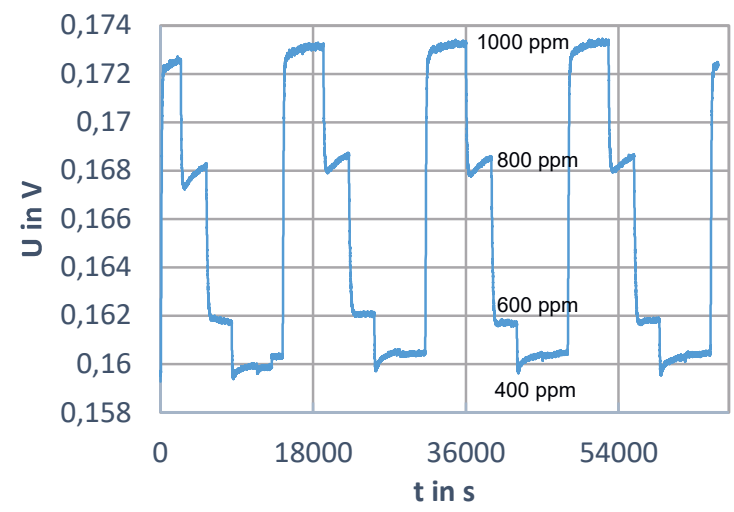

Abb. 11: Pd-beschichteter MEMS-Sensor beim Wechsel 1000 bis 400 ppm $\mathrm{H}_{2}$ in Luft

\section{Diskussion}

Die dargestellten Messergebnisse zeigen, dass die sensorischen Eigenschaften von $\mathrm{Pd}$ durch physikalische Eigenschaften des $\mathrm{Pd}-\mathrm{H}-$ Systems sehr gut erklärbar sind. Die Aufnahme von Wasserstoff durch Pd ist reversibel, sie folgt dem äußeren Partialdruck im Messgas (Sievertsches Gesetz). Der zunächst auf der Oberfläche adsorbierte Wasserstoff diffundiert in das Metallgitter, Triebkraft ist der Konzentrationsgradient, es stellt sich eine dem Phasengleichgewicht entsprechende Gleichgewichtskonzentration von $\mathrm{H}$-Atomen im Metallgitter ein. Die Transparenz der Pd-Dünnfilme entsteht mit Auftreten der $\beta$-Phase ab der Löslichkeitsgrenze der a-Phase. Der optische Sensor kann von daher sehr kleine Wasserstoff-Partialdrücke nicht nachweisen, da die $\alpha$-Phase metallisch opak ist. Der Übergang von $\alpha$ - zur $\beta$-Phase kann durch innere Spannungen im ultra-dünnen Pd/Au-Film in die eine oder andere Richtung gegenüber der Gleichgewichtslöslichkeitsgrenze, die aus dem 
Pd-H-Phasendiagramm [6] [8] bekannt ist, verschoben sein. Wie auch immer, wir können zeigen, dass mittels optischer Messungen in Transmission 1000 ppm Wasserstoff sicher nachgewiesen werden können. Die T90 Zeit beträgt für den optischen Sensor unter $30 \mathrm{~s}$.

Die Volumenänderung des Metallgitters kann durch MEMS-Systeme ebenfalls für sensorische Zwecke zum Nachweis $\mathrm{H}_{2}$ genutzt werden. Für Konzentrationen zwischen 1 vol\% und 100 vol\% beträgt die $T_{90}$ Zeit wenige Sekunden. Die Messungen sind reversibel und zeigen keine Drift. Eine Volumenänderung tritt auch bereits in der a-Phase auf, sodass mit der hier vorgestellten MEMS-Sensor-Struktur minimale Wasserstoffkonzentrationen von 100 ppm nachgewiesen werden können. Die $T_{90}$ Zeit für kleine Konzentrationen unter 1000 ppm beträgt jedoch mehrere Minuten. Ungewöhnlich ist die in Abbildung 11 sichtbare Drift der Messsignale bei der Messung kleiner Konzentrationen nach Einstellung des Messwertes. Diese Erscheinung ist scheinbar reversibel, da sie sich bei der zyklischen Messung der Wasserstoffbeladungen exakt wiederholt. Dies kann an den elastomachanischen Eigenschaften der MEMSStruktur liegen oder durch innere Spannungen im Metallfilm (Rekristallisation, Relaxation [6]) verursacht sein. Dies ist Gegenstand weiterer Untersuchungen bezüglich elasto-mechanisch angepasster MEMS-Pd-Dünnfilm-Strukturen zum Nachweis kleiner $\mathrm{H}_{2}$-Konzentrationen.

\section{Schlussfolgerungen}

Die hier dargestellten Pd-basierten optischen und MEMS Sensoren können sicher Wasserstoffkonzentrationen von 0,1 bis 100 vol\% messen und weisen Potenziale für neue Anwendungsfelder auf, da sie auf dem physikalischen Schalten von $\mathrm{Pd}$ bei Raumtemperatur beruhen. Sie haben keine Querempfindlichkeiten, sie messen $\mathrm{H}_{2-}$ Konzentrationen in $\mathrm{O}_{2}$-freier Umgebung und müssen nicht beheizt werden. Optische Sensoren sind zudem intrinsich sicher, da sie keine elektrischen Komponenten im zu überwachenden führen (Lichtleiterkabel). Pd-basierte MEMS Systeme haben im Konzentrationsbereich 1 -100 vol\% kurze Ansprechzeiten von wenigen Sekunden. Wir sehen von daher Anwendungspotenziale für die Einspeisung von regenrativ erzeugtem Wasserstoff in das Erdgasnetz. Pd-basierte optische und MEMS Sensoren erfüllen die ISO 26142 zur Kontrolle und zum Explosionsschutz von stationären Anlagen.

Weitere Einsatzgebiete sehen wir für die Kontrolle von Prozessen, bei denen in reduzierender Atmosphäre von bis zu 100 vol\% Wasserstoff gearbeitet werden muß, etwa bei der Herstellung von Reinstmetallen oder in der chemischen Verfahrenstechnik.

Potenziale weisen die hier vorgestellten PdMEMS-Sensoren für die Atemgasmesstechnik auf, wo verläßlich kleine $\mathrm{H}_{2}$-Konzentrationen unter 100 ppm gemessen werden müssen. Hier kommt es besonders auf die Querempfindlichkeitsfreiheit insbesondere gegenüber Ethanol und anderen Kohlenwasserstoffen an (Desinfektionsmittel in klinischer Umgebung). Die beschriebenen relativ langen Ansprechzeiten wären in diesem Anwendungsfeld akzeptabel, da keine Konzentrationen im Explosivbereich auftreten können. Sichere Messwerte könnten nach einigen Minuten ausgelesen werden.

\section{Dank}

Das Vorhaben wurde mit Mitteln des Bundesministeriums für Bildung und Forschung unter dem Förderkennzeichen $03 Z Z 0724$ gefördert.

\section{Literaturnachweis}

[1] T. Hübert, L. Boon-Brett, V. Palmisano, M. A. Bader, Developments in gas sensor technology for hydrogen safety, International Journal of hydrogen energy 39, 20474-20483 (2014); doi: 10.1016/j.ijhydene.2014.05.042.

[2] L. Boon-Brett, J. Bousek, G. Black, P. Moretto, P. Castello, T. Hübert, U. Banach, Identifying performance gaps in hydrogen safety sensor technology for automotive and stationary applications, International Journal of hydrogen energy 35, 373-384 (2010); doi: 10.1016/j.ijhydene.2009.10.064.

[3] Simon C Fleming, Evaluation of a handheld hydrogen monitor in the diagnosis of intestianl lactase deficiency, Ann Clin Biochem 27, 499-500 (1990).

[4] V. Palmisano, L. Boon-Brett, C. Bonato, F. Harskamp, W. J. Buttner, M. B. Post, R. Burgess, C. Rivkin, Evaluation of selectivity of commercial hydrogen sensors, International Journal of hydrogen energy 39, 20491-20496 (2014); doi: 10.1016/j.ijhydene.2014.03.251.

[5] Prabhu Soundarrajan and Frank Schweighardt: Hydrogen Sensing and Detection, In: Gupta, Ram B., ed., Hydrogen fuel: Production, transport, and storage. CRC; Taylor \& Francis, Boca Raton, Fla., London; 2009. 
[6] Astrid Pundt, Nanoskalige MetallWasserstoff-Systeme. Universitätsverlag Göttingen; 2005.

[7] A. Mandelis, J. A. Garcia, Sensors and Actuators B: Chemical 49, 258-267 (1998).

[8] F.D. Manchaster, A.San-Martin, and J.M. Pitre, The H-Pd (Hydrogen-Palladium) System, Journal of Phase Equilibria 15, 62-83 (1994).

[9] P. Fedtke, M. Wienecke, M.-C. Bunescu, M. Pietrzak, K. Deistung, E. Borchardt, Hydrogen sensor based on optical and electrical switching, Sensors and Actuators B: Chemical 100, 151-157 (2004); doi: 10.1016/j.snb.2003.12.062.

[10] Y. Oh, J. Hamagami, Y. Watanabe, M. Takata, A novel palladium thin film hydrogen-detector, J. Ceram. Soc. Jpn. 101, 605 (1993).

[11] Y. Liu, Y. Li, Enhanced sensitivity of transmission based optical fiber hydrogen sensor with multi-layer $\mathrm{Pd}-\mathrm{Y}$ alloy thin film, Sensors and Actuators B: Chemical 227, 178-184 (2016); doi: 10.1016/j.snb.2015.11.112.

[12] Y. Lim, Y. Lee, J.-I. Heo, H. Shin, Highly sensitive hydrogen gas sensor based on a suspended palladium/carbon nanowire fabricated via batch microfabrication processes, Sensors and Actuators B: Chemical 210, 218-224 (2015); doi: 10.1016/j.snb.2014.12.109.

[13] P.-G. Su, Y.-S. Chuang, Flexible H2 sensors fabricated by layer-by-layer selfassembly thin film of multi-walled carbon nanotubes and modified in situ with $\mathrm{Pd}$ nanoparticles, Sensors and Actuators B: Chemical 145, 521-526 (2010); doi: 10.1016/j.snb.2009.12.068.

[14] P. A. Pandey, N. R. Wilson, J. A. Covington, $\mathrm{Pd}$-doped reduced graphene oxide sensing films for $\mathrm{H} 2$ detection, Sensors and Actuators B: Chemical 183, 478-487 (2013); doi: 10.1016/j.snb.2013.03.089.

[15] J.-S. Noh, J. M. Lee, W. Lee, Lowdimensional palladium nanostructures for fast and reliable hydrogen gas detection, Sensors (Basel, Switzerland) 11, 825-851 (2011); doi: 10.3390/s110100825.

[16] G. Bramann, B. Zacharias, M. Wienecke, New optical hydrogen sensor with fast response time based on multilayer palladium-nickel-PTFE thin film for explosion-proof detection of high $\mathrm{H} 2$ concentrations of $1-100 \%$. In: Third European Workshop on Optical Fibre Sensors, 66191L. SPIE; 2007.
[17] D. R. Baselt, B. Fruhberger, E. Klaassen, S. Cemalovic, C. L. Britton, S. V. Patel, T. E. Mlsna, d. McCorkle, B. Warmack, Design and performance of a microcantilever-based hydrogen sensor, Sensors and Actuators B: Chemical 88, 120-131 (2003); doi: 10.1016/S09254005(02)00315-5.

[18] J. T. Gurusamy, G. Putrino, R. d. Jeffery, K.K.M.B. D. Silva, M. Martyniuk, A. Keating, L. Faraone, MEMS based hydrogen sensing with parts-per-billion resolution, Sensors and Actuators B: Chemical 281, 335-342 (2019); doi: 10.1016/j.snb.2018.07.118.

[19] Junmin Lee, Wooyoung Shim, Jin-Seo Noh, and Wooyoung Lee, Design Rules for Nanogap-Based Hydrogen Gas Sensors, ChemPhysChem 13, 1395-1403 (2012). 